\title{
Discriminating between the von Neumann and Lüders reduction rule
}

\author{
G. C. Hegerfeldt ${ }^{1,2}$ and R. Sala Mayato ${ }^{1,3}$ \\ ${ }^{1}$ Max Planck Institute for the Physics of Complex Systems, Nöthnitzer Str. 38 D-01187 Dresden, Germany \\ ${ }^{2}$ Institut für Theoretische Physik, Universität Göttingen, \\ Friedrich-Hund-Platz 1, D-37077 Göttingen, Germany \\ ${ }^{3}$ Departamento de Física Fundamental II and IUdEA, \\ Universidad de La Laguna, La Laguna, 38204, S/C de Tenerife, Spain
}

\begin{abstract}
Given an ensemble of systems in an unknown state, as well as an observable $\hat{A}$ and a physical apparatus which performs a measurement of $\hat{A}$ on the ensemble, whose detailed working is unknown ('black box'), how can one test whether the Lüders or von Neumann reduction rule applies?
\end{abstract}

PACS numbers: 03.65.-w; 03.65.Ta

\section{INTRODUCTION}

In his ground-breaking book [1] of 1932, von Neumann investigated the quantum mechanical measurement problem and formulated a rule how to obtain the state of an ensemble of physical systems after a measurement. This rule was later substantially modified by Lüders [2]. It is the Lüders reduction or projection rule that nowadays is mostly used. The Lüders rule states that after a selective measurement [3] of an observable $\hat{A}$ with discrete eigenvalues the subensemble of systems with the measurement result $a_{i}$ is in the (non-normalized, pure) state $\hat{P}_{i}|\psi\rangle$, where $\hat{P}_{i}$ is the (possibly multi-dimensional) projection operator onto the eigenspace of the eigenvalue $a_{i}$ and where $|\psi\rangle$ the state prior to the measurement. For an initial density matrix $\hat{\rho}$ one obtains $\hat{P}_{i} \hat{\rho} \hat{P}_{i}[4]$.

The von Neumann reduction rule assumes that in the case of degeneracy one measures a refinement $\hat{A}^{\prime}$ of $\hat{A}$, which commutes with $\hat{A}$ and which has only nondegenerate discrete eigenvalues [1] and thus lifts the degeneracy. Usually this results from consecutive measurements (for examples cf. e.g. [5] and Eq. (A5) of the Appendix below). Then $\hat{A}$ is a function of $\hat{A}^{\prime}$, $\hat{A}=f\left(\hat{A}^{\prime}\right)$, say, and from a measurement result $a^{\prime}$ of $\hat{A}^{\prime}$ for an individual system one obtains the corresponding result $a=f\left(a^{\prime}\right)$ for $\hat{A}$. As a generalization we introduce here the notion of a partial von Neumann type measurement which can also arise from consecutive measurements (cf. Appendix). One can choose a refinement which lifts the degeneracy of $\hat{A}$ only partially. Then still $\hat{A}=f\left(\hat{A}^{\prime}\right)$, but $\hat{A}^{\prime}$ may have some degenerate eigenvalues. Then a partial von Neumann type measurement is obtained by performing a Lüders type measurement of this observable $\hat{A}^{\prime}$. There are recent and important investigations of the state after more general measurements [6, 7], but we restrict ourselves to the above reduction rules.

In this paper we propose a simple three-step procedure, based on selective measurements, to test whether one deals with a Lüders type measurement of an observable $\hat{A}$ or not. We illustrate this for a particular measurement result, $a_{1}$ say. After the measurement the subensemble of systems with the result $a_{1}$ is selected and denoted by
$\mathcal{E}_{1}$. Then a refinement of $\hat{A}$, denoted by $\hat{\sigma}$, with discrete non-degenerate eigenvalues, is measured for each system of $\mathcal{E}_{1}$. Then on $\mathcal{E}_{1}$ one again measures $\hat{A}$ by means of the unknown apparatus and then again $\hat{\sigma}$. If for any system of $\mathcal{E}_{1}$ the result of the second measurement of $\hat{\sigma}$ differs from the first, one does not have a Lüders type measurement. If the results are the same for each system one chooses another, particular, refinement $\hat{\sigma}^{\prime}$ of $\hat{A}$ with non-degenerate eigenvalues and which does not commute with $\hat{\sigma}$ (i.e. only with $\hat{A}$ ). Then one proceeds as before, with $\hat{\sigma}^{\prime}$ instead of $\hat{\sigma}$. But now it turns out that one has a Lüders type measurement (on $\mathcal{E}_{1}$ ) if and only if for each system the two results of the $\hat{\sigma}^{\prime}$ measurements are the same.

The plan of the paper is as follows. In Section @ we show how the procedure works in the simple case of an observable with twofold degeneracy. In Section III the general case is treated. In Section IV the results are discussed. In the Appendix we give examples of Lüders, von Neumann, and partial von Neumann type measurements.

\section{TESTING THE TWOFOLD DEGENERATE CASE}

For greater transparency the procedure will first be explained for the example of the Appendix with two spins, $\hat{A}=\sigma_{1 z}+\sigma_{2 z}$. The eigenvalue $a_{1}=0$ of $\hat{A}$ is twofold degenerate. An as yet unknown apparatus performs a measurement of $\hat{A}$ on an ensemble $\mathcal{E}$. The apparatus can be assumed to perform a measurement of an observable $\hat{A}^{\prime}$, which is a possible trivial or nontrivial refinement of $\hat{A}$. We say that $\hat{A}^{\prime}$ is associated to the apparatus.

We assume that the result $a_{1}=0$ is found on a subensemble $\mathcal{E}_{1}$ of systems. In the two-dimensional eigenspace of the eigenvalue $a_{1}$ of $\hat{A}$ the as yet unknown observable $\hat{A}^{\prime}$, which commutes with $\hat{A}$, either has two non-degenerate eigenvalues or a single twofold degenerate eigenvalue. In the former case the apparatus performs a von Neumann measurement and in the latter a Lüders measurement.

Now we choose a refinement $\hat{\sigma}$ of $\hat{A}$ with nondegenerate eigenvalues. As an example, we take it to 
be diagonal in the basis $|++\rangle,|+-\rangle,|-+\rangle$, and $|--\rangle$, e.g.

$$
\hat{\sigma}=\sum_{i j= \pm}(2 i+j)|i j\rangle\langle i j|
$$

In the two-dimensional eigenspace of $\hat{A}$ for $a_{1}$ the eigenvalues and eigenvectors of $\hat{\sigma}$ are $s_{1}=1$ with $\left|s_{1}\right\rangle=|+-\rangle$ and $s_{2}=-1$ with $\left|s_{2}\right\rangle=|-+\rangle$. It may happen that, inadvertently and at this stage unknown to us, the chosen $\hat{\sigma}$ and the unknown operator $\hat{A}^{\prime}$ associated with the apparatus are jointly diagonal and commuting. This will bring a complication and will later require an additional step in the procedure. Now we proceed as follows.

(i) First a measurement of $\hat{\sigma}$ is performed on the subensemble $\mathcal{E}_{1}$. Since $\hat{\sigma}$ has only non-degenerate eigenvalues there is no difference between a von Neumann and a Lüders measurement of $\hat{\sigma}$ and the possible results are $s_{1}$ and $s_{2}$. If $s_{1}$ is found the subensemble of corresponding systems in $\mathcal{E}_{1}$ is denoted by $\mathcal{E}_{11}$, and after this measurement it is in the pure state $\left|s_{1}\right\rangle$. Similarly for $s_{2}$.

(ii) Now one lets the apparatus measure $\hat{A}$ on the systems of $\mathcal{E}_{1}$. Of course, the value found is again $a_{1}$ for each system. If the apparatus performs a Lüders measurement the state $\left|s_{i}\right\rangle$ of subensemble $\mathcal{E}_{1 i}$ is definitely not changed, while for a von Neumann measurement a change of the state $\left|s_{i}\right\rangle$ may or may not occur.

(iii) After this one again measures $\hat{\sigma}$ on $\mathcal{E}_{1}$. If one finds both $s_{1}$ and $s_{2}$ for systems in $\mathcal{E}_{11}$, then the state $\left|s_{1}\right\rangle$ has been changed and one concludes that the apparatus performs a von Neumann measurement. Similarly for $\mathcal{E}_{12}$.

If, on the other hand, one finds only $s_{1}$ on $\mathcal{E}_{11}$ this means that one of the projection operators in the decomposition of $\hat{A}^{\prime}$ leaves $\left|s_{1}\right\rangle$ invariant and that $\left|s_{1}\right\rangle$ is an eigenvector of $\hat{A}^{\prime}$. But then the orthogonal vector $\left|s_{2}\right\rangle$ is also an eigenvector of $\hat{A}^{\prime}$. Thus $\hat{A}^{\prime}$ and $\hat{\sigma}$ are diagonal in the same basis and commute. To find out if the above unknown projection operator of $\hat{A}^{\prime}$ is two-dimensional or not one now chooses another operator, $\hat{\sigma}^{\prime}$ say, which does not commute with $\hat{\sigma}$, e.g.

$$
\hat{\sigma}^{\prime}=\sigma_{1 z}+\sigma_{2 z}+\left(\vec{\sigma}_{1}+\vec{\sigma}_{2}\right)^{2} .
$$

The relevant eigenvalues are $s_{1}^{\prime}=4$ and $s_{2}^{\prime}=0$ with eigenvectors $\left|s_{1,2}^{\prime}\right\rangle=\left|\phi_{+,-}\right\rangle$from Eq. (A2), and they are not orthogonal to $\left|s_{1}\right\rangle$, with $\left\langle s_{1} \mid s_{1,2}^{\prime}\right\rangle \neq 0$. Now one proceeds as before for $\hat{\sigma}$. One first measures $\hat{\sigma}^{\prime}$ on the systems of $\mathcal{E}_{11}$, which is in the state $\left|s_{1}\right\rangle$, and denotes by $\mathcal{E}_{11}^{\prime}$ the subensemble of systems for which the value $s_{1}^{\prime}$ has been found. Then, on $\mathcal{E}_{11}^{\prime}$, which is in the state $\left|s_{1}^{\prime}\right\rangle$, one lets the apparatus perform a measurement of $\hat{A}$. This measurement again yields the value $a_{1}$, but it may or may not have changed the state of $\mathcal{E}_{11}^{\prime}$, depending on whether the apparatus performs a von Neumann or Lüders measurement. Then again $\hat{\sigma}^{\prime}$ is measured on $\mathcal{E}_{11}^{\prime}$. If both $s_{1}^{\prime}$ and $s_{2}^{\prime}$ appear as measurement results the state has been changed and therefore the apparatus performs a von Neumann measurement. If only the value $s_{1}^{\prime}$ appears then $\left|s_{1}^{\prime}\right\rangle$ is an eigenstate of $\hat{A}^{\prime}$, as is $\left|s_{1}\right\rangle$, from the above argument. From the non-orthogonality of $\left|s_{1}\right\rangle$ and $\left|s_{1}^{\prime}\right\rangle$ it follows that the corresponding eigenspace of $\hat{A}^{\prime}$ is twodimensional. Hence in this case the apparatus performs a Lüders measurement.

\section{THE GENERAL TEST}

In this section we describe the test procedure for Lüders vs. von Neumann for a general observable $\hat{A}$ with discrete, possibly degenerate, eigenvalues $a_{k}$. Corresponding orthogonal eigenvectors are denoted by $\left|a_{k}^{\alpha}\right\rangle$, $\alpha=1, \cdots, n_{k}$ so that the degeneracy is $n_{k}$. Then

$$
\begin{aligned}
\hat{A}_{k} & =\sum_{k, \alpha} a_{k}\left|a_{k}^{\alpha}\right\rangle\left\langle a_{k}^{\alpha}\right| \\
& \equiv \sum_{k} a_{k} \hat{P}_{k}
\end{aligned}
$$

where $\hat{P}_{k}$ is the projection operator onto the eigenspace of $a_{k}$. According to the Lüders rule the subensemble with the measurement result $a_{k}$ is described by

$$
\hat{P}_{k}|\psi\rangle
$$

in case of a pure initial state $|\psi\rangle$, and by

$$
\hat{P}_{k} \hat{\rho} \hat{P}_{k}
$$

in case of a mixed initial state $\hat{\rho}$. Both the norm squared and the trace give the probability of finding the value $a_{k}$. The complete ensemble is, directly after the measurement, described by the normalized density matrix

$$
\sum_{k} \hat{P}_{k} \hat{\rho} \hat{P}_{k}
$$

Now consider a refinement $\hat{A}^{\prime}$ of $\hat{A}$ which partially lifts the degeneracy of $\hat{A}$. Then $\hat{A}^{\prime}$ is of the form

$$
\begin{aligned}
& A^{\prime}=\sum_{k \beta} a_{k \beta}^{\prime} \hat{P}_{k}^{\beta} \\
& \hat{P}_{k}=\sum_{\beta=1}^{m_{k}} \hat{P}_{k}^{\beta}
\end{aligned}
$$

where the orthogonal projection operators $\hat{P}_{k}^{\beta}, \beta=$ $1 \cdots m_{k}$, are partial sums of $\left|a_{k}^{\alpha}\right\rangle\left\langle a_{k}^{\alpha}\right|$ for fixed $k$. Then $\hat{A}$ is a function of $\hat{A}^{\prime}, \hat{A}=f\left(\hat{A}^{\prime}\right)$, and $f\left(a_{k \beta}^{\prime}\right)=a_{k}$. A partial von Neumann measurement of $\hat{A}$ is obtained by a Lüders measurement of $\hat{A}^{\prime}$ where the apparatus is so programmed that its output is $f\left(a_{k \beta}^{\prime}\right)$ instead of $a_{k \beta}^{\prime}$. After the measurement the subensemble for which the output is $f\left(a_{k \beta}^{\prime}\right)=a_{k}$ is described instead of by Eq. (15) by the density matrix

$$
\sum_{\beta} \hat{P}_{k}^{\beta} \hat{\rho} \hat{P}_{k}^{\beta}
$$


and the complete ensemble by their sum over $k$. Note that if $m_{k}=1$ for all $k$, i.e. $\hat{P}_{k}^{\beta} \equiv \hat{P}_{k}^{1}=\hat{P}_{k}$, then one has a Lüders measurement, and if all $\hat{P}_{k}^{\beta}$ are one-dimensional projection operators one has a usual (i.e. not a partial) von Neumann measurement.

We now describe the test procedure Lüders vs. von Neumann for the general case and consider an ensemble $\mathcal{E}$ of systems with initial density matrix $\hat{\rho}$. As before we denote by $\hat{A}^{\prime}$ the observable associated to the unknown apparatus and consider the subensemble $\mathcal{E}_{1}$ of systems for which an eigenvalue $a_{1}$ of $\hat{A}$ has been found as the measurement result. The eigenvalue is $n_{1}$ fold degenerate. The subensemble $\mathcal{E}_{1}$ is described by the density matrix $\hat{\rho}_{1}$, with

$$
\hat{\rho}_{1}=\sum_{\beta=1}^{m_{1}} \hat{P}_{1}^{\beta} \hat{\rho} \hat{P}_{1}^{\beta}
$$

where the $\hat{P}_{k}^{\beta}$ are the unknown projection operators on eigenspaces of $\hat{A}^{\prime}$.

Let $\hat{\sigma}$ be an observable commuting with $\hat{A}$ and with discrete non-degenerate eigenvalues. The previous steps can now be adapted as follows.

(i) First one measures $\hat{\sigma}$ on the systems of $\mathcal{E}_{1}$. The eigenvalues of $\hat{\sigma}$ in the $a_{1}$ eigenspace are denoted by $s_{1}, \cdots, s_{n_{1}}$, with eigenstates $\left|s_{i}\right\rangle$. The subensemble of systems for which the result $s_{i}$ is found in the measurement will be denoted by $\mathcal{E}_{1 i}$. It can be described by the pure state $\left|s_{i}\right\rangle$.

(ii) Now one lets the apparatus perform a measurement of $\hat{A}$ on the systems of $\mathcal{E}_{1}$. The result is of course again $a_{1}$ and after the measurement the density matrix of the subensemble $\mathcal{E}_{1 i}$ is proportional to

$$
\sum_{\beta=1}^{m_{1}} \hat{P}_{1}^{\beta}\left|s_{i}\right\rangle\left\langle s_{i}\right| \hat{P}_{1}^{\beta}
$$

If $m_{1}=1$, i.e. if the apparatus performs a Lüders measurement in the $a_{1}$ eigenspace, this is the pure state $\left|s_{i}\right\rangle\left\langle s_{i}\right|$. Otherwise it is a mixed state.

(iii) After this, one again measures $\hat{\sigma}$ on $\mathcal{E}_{1}$. If for a system of a subensemble $\mathcal{E}_{1 i}$ this second measurement of $\hat{\sigma}$ gives a result different from $s_{i}$ one concludes from step (ii) that the apparatus has changed the state $\left|s_{i}\right\rangle$ and thus has not performed a Lüders measurement, but rather a (possibly partial) von Neumann measurement.

If, however, the result is always $s_{i}$ for each $\mathcal{E}_{1 i}$ then $\mathcal{E}_{1 i}$ remains in its state $\left|s_{i}\right\rangle$ after the measurement of $\hat{A}$ by the apparatus and hence this state is an eigenvector of $\hat{A}^{\prime}$. (If all $s_{i}$ appear as measurement results this implies that $\hat{A}^{\prime}$ and $\hat{\sigma}$ happen to commute on the $a_{1}$ eigenspace.)

In this case one chooses an additional observable $\hat{\sigma}^{\prime}$, with non-degenerate eigenvalues and which commutes with $\hat{A}$ but not with $\hat{\sigma}$ and which has the following special property. In the $a_{1}$ eigenspace the eigenvalues and eigenvectors of $\hat{\sigma}^{\prime}$ are denoted by $s_{i}^{\prime}$ and $\left|s_{i}^{\prime}\right\rangle$. The latter are connected to the eigenvectors $\left|s_{j}\right\rangle$ of $\hat{\sigma}$ by a unitary transformation, and one chooses $\hat{\sigma}^{\prime}$ in such a way that one has

$$
\left|s_{1}\right\rangle=\sum_{i=1}^{n_{1}} \gamma_{i}\left|s_{i}^{\prime}\right\rangle \quad \text { with } \gamma_{i} \neq 0 \quad \text { for all } i
$$

where $s_{1}$ is assumed to have occurred as a result in the measurement of $\hat{\sigma}$. Such a $\hat{\sigma}^{\prime}$ can always be found, and Eq. (12) is the key to distinguishing both types of measurements. On the systems of the subensemble $\mathcal{E}_{11}$ (which in this case has remained in the state $\left|s_{1}\right\rangle$ ) one then performs, with $\hat{\sigma}$ replaced by $\hat{\sigma}^{\prime}$, the steps (i)-(iii) . Since the transition probability $\left|\left\langle s_{1} \mid s_{j}^{\prime}\right\rangle\right|^{2}=\left|\gamma_{j}\right|^{2} \neq 0$, all eigenvalues $s_{i}^{\prime}$ appear as measurement results in the first measurement of $\hat{\sigma}^{\prime}$, and the associated subensembles $\mathcal{E}_{1 i}^{\prime}$ are in the state $\left|s_{i}^{\prime}\right\rangle$. Then, as the second step, one lets the apparatus perform a measurement of $\hat{A}$. In the third step $\hat{\sigma}^{\prime}$ is measured again on the systems of the subensemble $\mathcal{E}_{11}$. If for any system of $\mathcal{E}_{11}$ the result of the second measurement of $\hat{\sigma}^{\prime}$ differs from the first then the state has been changed by the apparatus and one has a (possibly partial) von Neumann measurement.

Otherwise, if for all systems of $\mathcal{E}_{11}$ the result of the second measurement of $\hat{\sigma}^{\prime}$ is the same as in the first, then the states $\left|s_{i}^{\prime}\right\rangle$ are not changed and hence are eigenvectors of $\hat{A}^{\prime}$, as is $\left|s_{1}\right\rangle$. Then all vectors in Eq. (12) are eigenvectors of $\hat{A}^{\prime}$. But this can only happen if they belong to the same eigenvalue since $\left|s_{1}\right\rangle$ is not orthogonal to any $\left|s_{i}^{\prime}\right\rangle$. This implies that the $a_{1}$ eigenspace of $\hat{A}$ is also an eigenspace of $\hat{A}^{\prime}$ and hence the apparatus performs a Lüders measurement of $a_{1}$ if for each system of the subensemble $\mathcal{E}_{11}$ the results of the first and second measurement of $\hat{\sigma}^{\prime}$ are the same.

\section{DISCUSSION}

In this paper the two forms of the reduction rule due to von Neumann and Lüders, also known as the projection postulate, have been discussed. The original formulation of von Neumann starts with an observable with discrete, possibly degenerate, eigenvalues, but then goes over to a refinement with non-degenerate eigenvalues, thus lifting the degeneracy. The projection operators are then one-dimensional and project onto the individual non-degenerate eigenvectors. Lüders, on the other hand, does not lift the degeneracy but uses projections onto eigenspaces of the original observable. The dimension of these eigenspaces are given by the degeneracy of the observable under consideration. In this paper we have also introduced an additional, sort of intermediary, reduction rule for which a refinement of the observable is used which lifts the degeneracy only partially and which may retain some degeneracy. We call the associated measurement a partial von Neumann measurement.

It has been shown here that all three forms of the reduction rule may appear quite naturally, depending on the realization of a particular measurement apparatus. 
Therefore all three forms have their own legitimacy, and one can not say that one is better than the other. Their applicability depends on the circumstances, i.e. the details of the measurement apparatus.

The main investigation of this paper focused on the following question. If a measurement apparatus for an observable is only known to obey one of the forms of the reduction rule of von Neumann and Lüders, but otherwise the details of the apparatus are not known how can one check whether the reduction has occurred by the Lüders rule or not? To this end we have proposed and studied a three-step procedure based on measurements of an auxiliary observable. The outcome of the latter measurements indicates the type of reduction.

It would be interesting if one could carry this investigation over to the more general types of measurements characterized in Ref. 6].

\section{Acknowledgements}

We thank J.G. Muga for discussions. We also acknowledge the kind hospitality of the Max Planck Institute for Complex Systems in Dresden, and funding of R. Sala Mayato by Ministerio de Ciencia e Innovación (Grant No. FIS2010-19998).

\section{Appendix A: Examples for different measurement schemes}

We consider an ensemble consisting of systems, each with two independent spins, $\vec{\sigma}_{1}$ and $\vec{\sigma}_{2}$, with $\vec{\sigma}$ the Pauli matrices. For the $z$ component of the total spin, $\sigma_{\mathrm{tot}, z}=$ $\sigma_{1 z}+\sigma_{2 z}$ the possible measurement results are $2,0,0$, -2 , with corresponding eigenvectors $|++\rangle,|+-\rangle,|-+\rangle$, and $|--\rangle$.

Now, in case of a Lüders type measurement, if the initial state of the ensemble is a pure state $|\psi\rangle$ then after the measurement the respective subensembles are given by the pure states $\hat{P}_{i}|\psi\rangle, i=1,0,-1$. Here

$$
\hat{P}_{1}=\hat{P}_{|++\rangle}, \hat{P}_{0}=\left\{\hat{P}_{|+-\rangle}+\hat{P}_{|-+\rangle}\right\}, \hat{P}_{-1}=\hat{P}_{|--\rangle}
$$

where $\hat{P}_{|\phi\rangle} \equiv|\phi\rangle\langle\phi|$. The total ensemble is then described after this measurement by a density matrix given by $\sum_{i} \hat{P}_{i}|\psi\rangle\langle\psi| \hat{P}_{i}$. Similarly for an initial density matrix instead of a pure state.

Following von Neumann, instead of $\sigma_{\text {tot }, z}$ one can measure a refinement of $\sigma_{\mathrm{tot}, z}$ with non-degenerate eigenvalues, e.g. the observable $\hat{A}^{\prime} \equiv \sigma_{\text {tot }, z}+\left(\vec{\sigma}_{\text {tot }}\right)^{2}$, which lifts the degeneracy of $\sigma_{\text {tot }, z}$. From this one obtains an indirect measurement of $\sigma_{\text {tot }, z}$ as follows. The eigenvectors of $\hat{A}^{\prime}$ are

$$
\begin{array}{ll}
|++\rangle, \quad\left|\phi_{+}\right\rangle \equiv\{|+-\rangle+|-+\rangle\} / \sqrt{2}, \\
|--\rangle, \quad\left|\phi_{-}\right\rangle \equiv\{|+-\rangle-|-+\rangle\} / \sqrt{2},
\end{array}
$$

with respective eigenvalues $a^{\prime}=6,4,2$ and 0 . If one defines the function $f(x)=-\frac{8}{3} x+x^{2}-\frac{1}{12} x^{3}$ then $f(6)=$ $2, f(4)=0, f(2)=-2$ and $f(0)=0$, and $f\left(\hat{A}^{\prime}\right)=\sigma_{\text {tot }, z}$. This is either checked directly by insertion of $\hat{A}^{\prime}$ or by applying $f\left(\hat{A}^{\prime}\right)$ to the eigenvectors of $\hat{A}^{\prime}$. Therefore, if the result of an $\hat{A}^{\prime}$ measurement on a system is $a^{\prime}$, then one knows that $\sigma_{\text {tot }, z}$ has the values $f\left(a^{\prime}\right)$. In contrast to the previous Lüders measurement, now the subensemble with the result 0 for $\sigma_{\text {tot, } z}$ is in a mixed state, given by the density matrix

$$
\hat{P}_{\left|\phi_{+}\right\rangle}|\psi\rangle\left\langle\psi\left|\hat{P}_{\left|\phi_{+}\right\rangle}+\hat{P}_{\left|\phi_{-}\right\rangle}\right| \psi\right\rangle\langle\psi| \hat{P}_{\left|\phi_{-}\right\rangle} .
$$

The complete ensemble has now the density matrix

$$
\begin{aligned}
& \hat{P}_{|++\rangle}|\psi\rangle\left\langle\psi\left|\hat{P}_{|++\rangle}+\hat{P}_{\left|\phi_{+}\right\rangle}\right| \psi\right\rangle\langle\psi| \hat{P}_{\left|\phi_{+}\right\rangle} \\
& +\hat{P}_{\left|\phi_{-}\right\rangle}|\psi\rangle\left\langle\psi\left|\hat{P}_{\left|\phi_{-}\right\rangle}+\hat{P}_{|--\rangle}\right| \psi\right\rangle\langle\psi| \hat{P}_{|--\rangle} .
\end{aligned}
$$

For consecutive measurements von Neumann type measurements appear quite naturally. If the two spins are spatially sufficiently separated one can measure them individually, e.g. first a Lüders measurement of $\sigma_{1 z}$ and then immediately afterwards of $\sigma_{2 z}[5]$. This also provides a measurement of $\sigma_{\text {tot }, z}$. In this case, the possible individual measurement results are,,,+++--+-- , and after the measurement the corresponding subensembles are obviously in the states $|++\rangle, \cdots,|--\rangle$. If the initial state of the ensemble is a pure state $|\psi\rangle$ then after the measurement its state is given by the density matrix

$$
\sum_{i, j= \pm} \hat{P}_{|i j\rangle}|\psi\rangle\langle\psi| \hat{P}_{|i j\rangle}
$$

and similarly for an initial density matrix. It is apparent that this consecutive measurement amounts to a particular von Neumann measurement, but with a resulting density matrix which differs from the previous one in Eq. (A4). The measurement is equivalent to a separate measurement of the projection operators $\hat{P}_{|i j\rangle}$ or, equivalently, of an observable of the form $\hat{A}^{\prime}=\sum a_{i j}^{\prime} \hat{P}_{|i j\rangle}$ with pairwise different $a_{i j}^{\prime}$ 's. This observable $\hat{A}^{\prime}$ can be taken to be associated with the apparatus measuring the individual spins separately.

We now consider an ensemble consisting of systems each with three independent spins, $\vec{\sigma}_{1}, \vec{\sigma}_{2}$, and $\vec{\sigma}_{3}$, and the observable $\hat{A}=\sigma_{1 z}+\sigma_{2 z}$. One can now consecutively measure $\sigma_{1 z}$ and $\sigma_{2 z}$, as before. Then with three independent spins, this gives a partial von Neumann measurement of $\sigma_{1 z}+\sigma_{2 z}$. If one measured all three spins consecutively this would resolve the degeneracy and lead to an ordinary von Neumann measurement. 
[1] J. von Neumann, Mathematische Grundlagen der Quantenmechanik, (Springer, Berlin, 1932). English translation: J. von Neumann, Mathematical Foundations of Quantum Mechanics, Chapter V.1 (Princeton University Press, Princeton, 1955)

[2] G. Lüders, Ann. Phys. (Leipzig) 6(8), 322 (1951). English translation: G. Lüders, Ann. Phys. (Leipzig) 15, 663 (2006) (translated and discussion added by K.A. Kirkpatrick). In a private communication to one of us (G.C.H), Lüders $(1920$ - 1995) stressed the provisional character of the rule, provisional until a better understanding of the actual measurement process was achieved.

[3] A selective measurement is a measurement procedure in which the result for each individual system is recorded.

[4] The Lüders rule can be derived under the assumption of repeatability and minimal disturbance caused by the measurement, see G. Ludwig, Z. Phys. 135, 483 (1953); G. Ludwig, Die Grundlagen der Quantenmechanik, (Springer, Berlin, 1954). In this context it is noteworthy that Dirac, in the first edition of his famous book, defines observations causing minimal disturbance which seem closely related to the Lüders rule, see P.A.M. Dirac, The Principles of Quantum Mechanics, 1st Ed., p. 49 (Clarendon, Oxford, 1930). Curiously though, in later editions this passage has been omitted.

[5] R. Sala Mayato and J.G. Muga, Phys. Lett. A 375, 3167 (2011)

[6] E.G. Beltrametti, G. Cassinelli, and P.J. Lahti, J. Math Phys. 31, 91 (1990);

[7] M. Ozawa, J. Math. Phys. 25, 79 (1984); W.M. de Muynck, J. Phys. A: Math. Gen. 31, 431 (1998); P. Busch and P. Lahti, in Compendium of Quantum Physics, D. Greenberger, K. Hentschel and F. Weinert, Editors, (Springer, Berlin, 2009); T. Heinosaari, M.A. Jivulescu, D. Reitzner, and M. Ziman, Phys. Rev. A 82, 032328 (2010); K. Kraus, States, Effects, and Operations (Springer, Berlin, 1983); A. Khrennikov, J. Math. Phys. 49, 052102 (2008). 